\title{
Transportation Barriers and Health-Related Quality of Life in a Sample of Middle-Aged and Older Adults Living with HIV in the Deep South
}

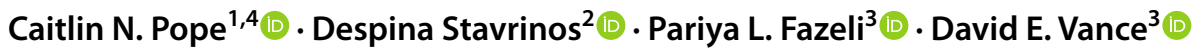

Accepted: 14 December 2021 / Published online: 23 January 2022

(c) The Author(s), under exclusive licence to Springer Science+Business Media, LLC, part of Springer Nature 2021

\begin{abstract}
Access to reliable transportation is a social determinant of health imperative for disease management for those aging with HIV/AIDS. To what degree transportation barriers are associated with health-related quality of life (HRQOL) in middleaged and older people living with HIV (PWH) in the Deep South region of the United States is presently unknown. PWH ( $n=261$, age range $=39$ to 73 years old, $80.1 \%$ African American, $64.4 \%$ male) were recruited from an academic medical center in the Deep South. Variables included sociodemographics, HIV characteristics, depressive symptoms, HRQOL, and perceived transportation barriers. Spearman rho correlations and linear regressions accounting for covariates were conducted. After accounting for covariates, greater perceived transportation barriers were associated with worse health perceptions, pain, social functioning, health distress, and health transitions. Access to reliable transportation is a key factor in improving health for PWH. Considerations for healthcare and traffic safety are discussed.
\end{abstract}

Keywords HIV/AIDS · Quality of life · Health status · Aging · Transportation

\section{Introduction}

Since the beginning of the HIV/AIDS epidemic in 1981, the burden of HIV/AIDS in the United States (U.S.) has shifted from large metropolitan areas in the Northeast and West coast to Southern states [1]. As of 2018, Southern states accounted for $51 \%$ of the new diagnoses in the U.S. and dependent areas and for almost half (47\%) of deaths within people with HIV (PWH) $[2,3]$. Within the South, $82 \%$ of diagnoses during 2012-2017 were found within the Deep South [4], defined by nine states: Alabama, Florida, Georgia, Louisiana, Mississippi, North Carolina, South Carolina, Tennessee, and Texas [5]. Demographic characteristics of

Caitlin N. Pope

Caitlin.Pope@uky.edu

1 Department of Health, Behavior \& Society, University of Kentucky, Lexington, KY, USA

2 Department of Psychology, University of Alabama at Birmingham, Birmingham, AL, USA

3 School of Nursing, University of Alabama at Birmingham, Birmingham, AL, USA

4 Graduate Center for Gerontology, 725 Rose St. Suite 401 Multidisciplinary Sciences Building, Lexington, KY 40536, USA
PWH in the Deep South show higher and disproportionate diagnosis rates for those of Black race and Hispanic ethnicity, men having sex with men (MSM), and individuals of lower socioeconomic status $[2,4,5]$. This is of great public health concern in terms of adequate access to healthcare as Southern states also have some of the highest rates of poverty, lowest median household incomes, and highest rates of medically uninsured individuals compared to other U.S. regions compounded with high rates of chronic disease such as cardiovascular disease and diabetes [5]. In addition to systemic barriers that may prohibit access to healthcare, individuals living in the Deep South may also face geographical and logistical barriers such as living in rural areas or having lack of access to adequate public transportation, even within urban areas, when compared to other U.S. regions [4-9].

Access to adequate transportation is a social determinant of health that is frequently overlooked in aging and public health research, as previous transportation-related studies have primarily focused on motor vehicle safety outcomes and indicators of driving abilities within aging samples [10]. While not uniquely a problem for PWH, previous research has shown a lack of adequate transportation has direct impacts on health-related outcomes in samples of adults living with chronic disease [7], patient retention in cardiac rehabilitation programs [11], access to care for those with 
traumatic brain injury (TBI) [12], and in U.S. Medicare beneficiaries [13], to name a few. Importantly, a lack of access to adequate transportation is not a newly discovered barrier for PWH. Heckman et al. designed the Barriers to Care Scale for PWH and found that individuals who lived in rural areas in Wisconsin reported more barriers to care than those who lived in urban areas [14]. Transportation and geography along with medical and psychological resources, community stigma, and personal resources were included in the scale. When specifically examining samples from the Deep South, Rief et al. surveyed HIV case managers in both rural and urban counties and areas in North Carolina and found that $30 \%$ of urban and $58 \%$ of rural case managers reported their patients having to travel long distances to obtain essential HIV care [15]. Additionally, Kempf et al. conducted qualitative focus groups with African-American women living in rural Alabama and found that transportation was reported as a major barrier to retention in HIV medical care, yet when transportation vouchers or transportation scheduling was provided by the clinic, this barrier was eliminated [6].

When assessing the association between transportation barriers and health outcomes in HIV/AIDS, most frequently studied in the literature is how transportation barriers impact HIV treatment outcomes such as HIV care retention [9] and delays to HIV care initiation [16]. Yehia et al. conducted semi-structured interviews with 51 PWH from urban Ryan White-funded clinics in Philadelphia and found a commonly reported barrier to retention in HIV care included expensive and unreliable transportation [9]. Within the same sample, those who took advantage of transportation services such as free bus/subway passes and van services reported these services facilitated their retention to HIV care [9]. Additionally, Lopes et al. found in a sample of 1,396 patients from the UNC CFAR HIV Clinical Cohort, that rural patients in comparison to urban patients were more likely to reside at longer distances from HIV clinics, were older in age, were more likely to be coinfected with the hepatitis $\mathrm{C}$ virus, and had lower CD4+T cell counts at HIV care initiation, yet intercorrelations between these indicators were not assessed [16].

Less known in the context of HIV is how transportation barriers are associated with other key health-related outcomes that are indicators of well-being and contribute to a more holistic approach to health promotion, such as healthrelated quality of life (HRQOL). HRQOL is operationally defined as how an individual or a group perceive their physical and mental health over a defined period of time [17]. Previous research in samples of PWH have found lower HRQOL for women compared to men [18], in individuals with lower socioeconomic status (measured by income, education, and employment status) [19], in individuals with a higher viral load [20], and in individuals with a diagnosis of lifetime major depressive disorder [21]. When specifically examining how HRQOL may be associated with transportation barriers, the literature is sparse in both PWH and nonHIV samples. Erskine et al. found a clinically meaningful decline in physical HRQOL and mental HRQOL for those who reported a transportation barrier to healthcare access in a cohort of Acute Coronary Syndrome survivors [22]. To date, there is a lack of literature connecting transportation barriers to HRQOL in samples of middle-aged and older PWH which may have a direct impact on aging trajectories and health promotion within this at-risk clinical group.

Given the dearth of literature on transportation barriers and how they may contribute to HRQOL, especially in samples of aging PWH, this study aims to 1) describe perceived transportation barriers and contributors to these barriers in a sample of middle-aged and older PWH in the Deep South, and 2) assess the association between perceived transportation barriers and contributors with health outcomes (CD4+T cell count, viral load, medication adherence, and depressive symptomology) and HRQOL. We hypothesized greater perceived transportation barriers would be associated with worse health outcomes and lower HRQOL in a sample of aging PWH in the Deep South.

\section{Methods}

\section{Participants and Procedure}

Participants included 261 middle-aged and older PWH enrolled in the baseline visit of a longitudinal study on neurocognitive aging. See Vance et al. for more detail on the parent study protocol [23]. Middle-aged and older individuals (40+ years) were targeted for recruitment from an academic medical center in the Deep South. Inclusion criteria included being (a) 40+ years old, (b) diagnosed with HIV/ AIDS for at least 1 year, (c) not diagnosed with a significant neuromedical condition (e.g., Alzheimer's disease, schizophrenia, traumatic brain injury); and (d) English-speaking. The procedure was reviewed and approved by the University of Alabama at Birmingham's Institutional Review Board and all participants provided written informed consent and were compensated for participating. PWH were screened for eligibility and recruited from 2016 to 2018.

\section{Measures}

\section{Demographics}

PWH self-reported sociodemographic information included age (measured in years), gender ( $0=$ "female", $1=$ "male"), race $(0=$ "White/Caucasian", $1=$ "Non-White" comprised of African American, Hispanic, Native American, and other races), education (measured in years), and 
annual household income before taxes $(1=" \$ 0-10,000 "$, $2=" \$ 10,001-20,000 ", 3=" \$ 20,001-30,000 "$, up until $\$ 100,001$ and above). Residence ZIP codes were coded using the 2010 Rural-Urban Commuting Area (RUCA) codes data set, which provide updated data from the 2010 decennial census and the 2006-2010 American Community Survey [24]. RUCA codes define urban (metropolitan areas, micropolitan areas, and small towns) and rural areas and clusters. The entire sample resided in an urban area or cluster with the majority $(97.3 \%, n=254)$ in metropolitan areas.

\section{HIV Characteristics}

Lab values were obtained from clinic records for current and nadir CD4+T cell counts, viral load, and if the individual was currently taking antiretroviral therapy (ART). PWH self-reported HIV duration in years since diagnosis along with medication adherence using the three-question Medication Adherence Questionnaire [25]. This questionnaire, which has been previously validated in an HIV sample [25], assessed HIV medication adherence by days taken, frequency, and rating over the last 30 days. Questionnaire answers were presented on Likert-type scales and were summed together to give an overall sum score of selfreported medication adherence. Summed scores could range from 3 to $17(\alpha=0.84)$, with higher scores indicating better medication adherence.

\section{Depressive Symptoms}

Depressive symptoms were measured using the Center for Epidemiologic Studies Depression Scale-Revised (CES-D) [26]. The measure contains 20 questions answered on Likert-type scales to assess feelings or behaviors over the past week. After applying reverse coding to positively framed questions, the answers were summed together to give an overall sum score of depressive symptoms. Summed scores could range from 0 to $60(\alpha=0.88)$, with higher scores indicating greater depressive symptoms and a score of 16 or greater reflecting individuals at risk for clinical depression [27].

\section{HRQOL}

HRQOL over the previous 4 weeks was measured using the Medical Outcomes Study Health Survey (MOS-HIV) [28]. The MOS-HIV has been widely used to study perceptions of physical and mental health in HIV/AIDS samples with generally good internal consistency reported across studies [29]. The questionnaire contained 35 questions, answered with either Likert-type or binary scales across 11 health domains: general health perceptions, pain, physical functioning, role functioning, social functioning, cognitive functioning, energy/fatigue, mental health, health distress, health transition, and quality of life. After applying reverse coding to negatively framed questions, the answers were summed into their retrospective domains based on previous psychometric evaluations [30, 31]. The domain scores were then linearly transformed to a 100-point scale with higher scores indicating better perceptions of health in the specific health domain.

\section{Perceived Transportation Barriers}

Given the breadth and diversity of transportation barriers, two questionnaires assessed perceived transportation barriers and contributors. Transportation usage was assessed using a 16-item, questionnaire created for the study which asked questions regarding how individuals moved around in their environment either by personally owned vehicles or public transportation and how transportation may impact health-related outcomes. Specifically, it assessed whether the individual had ever cancelled a healthcare appointment because of lack of transportation ( $0=$ "no", $1=$ "yes"), how often the individual planned healthcare appointments around transportation needs (Likert-type, $1=$ "never" to 5 "always"), and if adequate transportation improved their quality of life (Likert-type, 1 = "strongly agree" to $5=$ "strongly disagree"). Transportation barriers were measured using one item from the Barriers to Care Scale (BACS) [14]. Using a four-point Likert-type scale ( $1=$ "no problem at all" to $4=$ "major problem"), the question assessed how much of a problem it was for individuals to access the care, services, or opportunities they wished to obtain because of a lack of transportation.

\section{Data Analysis}

Descriptive characteristics were assessed for the entire sample based on the variable measurement. Spearman Rho correlations were conducted to assess the intercorrelations between demographics, perceived transportation barriers and contributors, depressive symptoms, and health outcomes. A series of hierarchical linear regressions were conducted for each HRQOL health domain with the first step including HIV characteristics (HIV duration, CD4+T cell count, viral load), the second step including sociodemographic characteristics (race, annual household income before taxes) and depressive symptoms, and the third step including transportation barriers to care (the single BACS item).

Regarding missing data, the largest amount of missing data was seen for HIV characteristic clinic data such as CD4+T cell count (missing $n=76$ ) and viral load (measured continuously; missing $n=47$ ). Clinic values were not obtained for participants who had not had a recently drawn lab value within 3 months before or after the study baseline 
visit. Missing data analyses revealed that those with missing CD4+T cell count and viral load were not statistically different on demographic characteristics (age, gender, education, annual household income before taxes), depressive symptoms, HIV characteristics (HIV duration in years since diagnosis, nadir CD4+T cell count, and self-reported medication adherence), or perceived transportation barriers (all $p$ values $>0.05$ ). All possible cases were used for descriptives and intercorrelations. The final analytic sample for regression models included $178 \mathrm{PWH}$. All analyses were conducted using SPSS 27 [32] with statistical significance denoted by $p<0.05$.

\section{Results}

Sample demographics are provided in Table 1. The total sample was on average 51.10 years old $(S D=6.78$ years, range $=39$ to 73$), 64.4 \%(n=168)$ of the sample identified as being male gender, and $80.1 \%(n=209)$ identified as being African American. Years of formal education ranged from approximately 7 to 20 years $(M=12.54$ years, $S D=2.24$ years) and most of the sample reported an annual household income before taxes of $\$ 20,000$ or less $(n=206$, $78.9 \%$ ). Half of the sample were at high risk for clinical depression as indicated by a 16 or higher on the CES-D $(n=133,51.0 \%)$ [26]. The estimated median duration of HIV since diagnosis for the sample was 18 years $[\mathrm{IQR}=10.8$, 24.0]. When assessing clinical data, the majority of $\mathrm{PWH}$ $(n=231,94.7 \%)$ with complete clinical data were currently taking ART and over half of the sample had undetectable viral load $(n=142,66.4 \%)$. The sample's current median $\mathrm{CD} 4+\mathrm{T}$ cell count was 636.0 cells $/ \mathrm{mm}^{3}[\mathrm{IQR}=382.0$ cells/ $\mathrm{mm}^{3}, 882.0$ cells $\left./ \mathrm{mm}^{3}\right]$.

Regarding HRQOL, on average most health domain scores ranged between scores of 60 and 80 (out of a total score of 100) depending on the domain (see Table 2). The lowest average domain score was seen for the energy/fatigue domain $(M=63.89, S D=18.83)$ and the highest average domain score was the role functioning domain $(M=77.97$, $S D=22.22$ ). This suggests that the current sample of PWH reported more frequent feelings of being worn out or tired, yet still reported being able to do their job or work around the house over the past four weeks, regardless of their health. When assessing perceived transportation barriers and contributors (see Table 3), only 63.6\% $(n=166)$ of the total sample had a current and valid state-issued driver's license and $67.8 \%(n=177)$ owned or had access to a vehicle. Over one-third of the sample $(n=98)$ reported having to cancel or miss a healthcare appointment because of lack of transportation. Additionally, over half of the sample ( $n=160,61.3 \%)$ reported, to some degree (ranging from 'rarely' to 'always'), having to frequently plan healthcare appointments around
Table 1 Sample characteristics (total $n=261$ )

\begin{tabular}{ll}
\hline Variable & $\begin{array}{l}\text { M (SD) or } \%(n) \text { or } \\
\text { median [IQR] }\end{array}$ \\
\hline Age (years) & $51.10(6.78)$ \\
Education (years) & $12.54(2.24)$ \\
CES-D total score & $17.95(11.00)$ \\
Gender (male) & $64.4 \%(168)$ \\
Race & \\
African American & $80.1 \%(209)$ \\
White & $17.2 \%(45)$ \\
Other & $2.7 \%(7)$ \\
Work status (not employed) & $78.2 \%(204)$ \\
Disabled (yes) & $60.5 \%(158)$ \\
Income & \\
$0-10,000$ & $51.3 \%(134)$ \\
10,001-20,000 & $27.6 \%(72)$ \\
20,001-30,000 & $10.0 \%(26)$ \\
$>30,000$ & $11.1 \%(29)$ \\
HIV characteristics & \\
HIV duration (years) ${ }^{\mathrm{b}}$ & $18.0[10.8,24.0]$ \\
CD4+T cell count $\left(\mathrm{cells} / \mathrm{mm}^{3}\right)^{\mathrm{c}}$ & $636.0[382.0,882.0]$ \\
Nadir CD4+T cell count $\left(\mathrm{cells}^{\mathrm{a}} / \mathrm{mm}^{3}\right)^{\mathrm{d}}$ & $192.0[25.8,465.0]$ \\
${\text { Viral load (copies } / \mathrm{mL}^{\mathrm{e}}}^{\mathrm{e}}$ & $19.0[19.0,40.5]$ \\
Detectable viral load & $33.6 \%(72)$ \\
ART (currently taking) & $\mathrm{f}$ \\
\hline
\end{tabular}

$A R T$ antiretroviral therapy, $C E S-D$ Center for Epidemiologic Studies depression scale, $I Q R$ interquartile range, $M$ mean, $S D$ standard deviation

${ }^{\mathrm{a}}$ Annual household income before taxes

${ }^{\mathrm{b}} n$ (total for this measure) $=254$

${ }^{\mathrm{c}} n$ (total for this measure) $=185 ; \mathrm{CD} 4+\mathrm{T}$ cell count was measured within a 3-month period (prior or after) the baseline visit

${ }^{\mathrm{d}} n$ (total for this measure $)=238$

${ }^{\mathrm{e}} n$ (total for this measure $)=214$; viral load was measured within a 3 -month period (prior or after) the baseline visit

${ }^{\mathrm{f}} n$ (total for this measure) $=244$

their transportation needs. When asked if adequate transportation improved their quality of life, $88.5 \%$ of the sample $(n=231)$ reported that they either agreed or strongly agreed with the statement. Distribution assessment of how much of a problem an individual had with accessing the care, services, or opportunities they wished to obtain because of a lack of transportation (i.e., the BACS item) showed that $67.4 \%(n=176)$ reported no problem, $13.4 \%(n=35)$ reported very slight problems, $13.4 \%(n=35)$ somewhat of a problem, and $5.7 \%(n=15)$ reported it as a major problem.

Spearman Rho intercorrelations on the associations between demographics, perceived transportation barriers, depressive symptoms, and health outcomes revealed that being of a non-White race and having fewer years of education was associated with having a lower annual household 
Table 2 HRQOL health domain scores measured with the Medical Outcome Study Health Survey (MOS-HIV)

\begin{tabular}{ll}
\hline Domains & M (SD) \\
\hline General health perceptions & $64.78(21.43)$ \\
Pain & $65.10(23.27)$ \\
Physical functioning & $74.29(18.79)$ \\
Role functioning & $77.97(22.22)$ \\
Social functioning & $75.10(24.75)$ \\
Cognitive functioning & $74.90(20.02)$ \\
Energy/fatigue & $63.89(18.83)$ \\
Mental health & $74.23(18.04)$ \\
Health distress & $75.48(23.17)$ \\
Health transition & $73.10(20.19)$ \\
Quality of life & $74.02(19.24)$ \\
\hline
\end{tabular}

$H R Q O L$ health-related quality of life, $M$ mean, $S D$ standard deviation HRQOL scores were transformed to a scale ranging from 0 to 100 , with high scores indicated better perceptions of health in the specific health domain

income, not owning or having access to a vehicle, having to more frequently plan healthcare appointments around transportation needs, and having more perceived transportation barriers (see Table 4). Additionally, having a lower annual household income was associated with not owning or having access to a vehicle $\left(r_{s}=0.28, p<0.001\right)$, having cancelled or missed a healthcare appointment because of lack of transportation $\left(r_{s}=-0.25, p<0.001\right)$, more frequent planning of healthcare appointments around transportation needs $\left(r_{s}=-0.31, p<0.001\right)$, greater perceived transportation barriers $\left(r_{s}=-0.18, p=0.004\right)$, poorer medication adherence $\left(r_{s}=0.13, p=0.040\right)$, and greater depressive symptoms $\left(r_{s}=-0.20, p=0.001\right)$. Lastly, greater perceived transportation barriers were associated with not owning or having access to a vehicle $\left(r_{s}=-0.28, p<0.001\right)$, having ever cancelled a healthcare appointment because of lack of transportation $\left(r_{s}=0.39, p<0.001\right)$, having to frequently plan healthcare appointments around transportation needs $\left(r_{s}=0.35, p<0.001\right)$, poorer medication adherence $\left(r_{s}=-0.18, p=0.006\right)$, greater depressive symptoms $\left(r_{s}=-0.25, p<0.001\right)$, and higher viral load $\left(r_{s}=0.17\right.$, $p=0.016)$.

Linear regressions with HRQOL health domains showed that after accounting for HIV duration, $\mathrm{CD} 4+\mathrm{T}$ cell count, viral load amount, race, annual household income, and depressive symptoms, perceived transportation barriers were independently associated with the following HRQOL domains: general health perceptions, pain, social functioning, health distress, and health transitions (see Table 5, the other HRQOL domains to which perceived transportation

Table 3 Perceived transportation barriers and contributors to those barriers (total $n=261$ )

\begin{tabular}{|c|c|}
\hline Variable & $\%(n)$ \\
\hline Do you have a current and valid driver's license? (yes) & $63.6(166)$ \\
\hline Do you currently drive regardless of having a current and valid driver's license? (yes) & $81.6(213)$ \\
\hline Do you own a car or have access to a car? (yes) & $67.8(177)$ \\
\hline Have you ever cancelled or not gone to a healthcare appointment because of lack of transportation? (yes) & $37.5(98)$ \\
\hline \multicolumn{2}{|l|}{ How often do you have to plan healthcare appointments around your transportation needs? } \\
\hline Never & $38.7(101)$ \\
\hline Rarely & $14.6(38)$ \\
\hline Sometimes & $17.6(46)$ \\
\hline Often & $7.7(20)$ \\
\hline Always & $21.5(56)$ \\
\hline \multicolumn{2}{|l|}{ To what degree do you agree with this statement: "Adequate transportation improves my quality of life." } \\
\hline Strongly disagree & $1.1(3)$ \\
\hline Disagree & $1.1(3)$ \\
\hline Neither agree nor disagree & $9.2(24)$ \\
\hline Agree & $19.5(51)$ \\
\hline Strongly Agree & $69.0(180)$ \\
\hline \multicolumn{2}{|c|}{$\begin{array}{l}\text { To what extent does the lack of transportation to access the services you need make it difficult for you to receive the care, services, } \\
\text { or opportunities you wish to obtain? }\end{array}$} \\
\hline No problem at all & $67.4(176)$ \\
\hline Very slight problem & $13.4(35)$ \\
\hline Somewhat a problem & $13.4(35)$ \\
\hline Major problem & $5.7(15)$ \\
\hline
\end{tabular}

${ }^{a}$ Measured with the barriers to care scale 


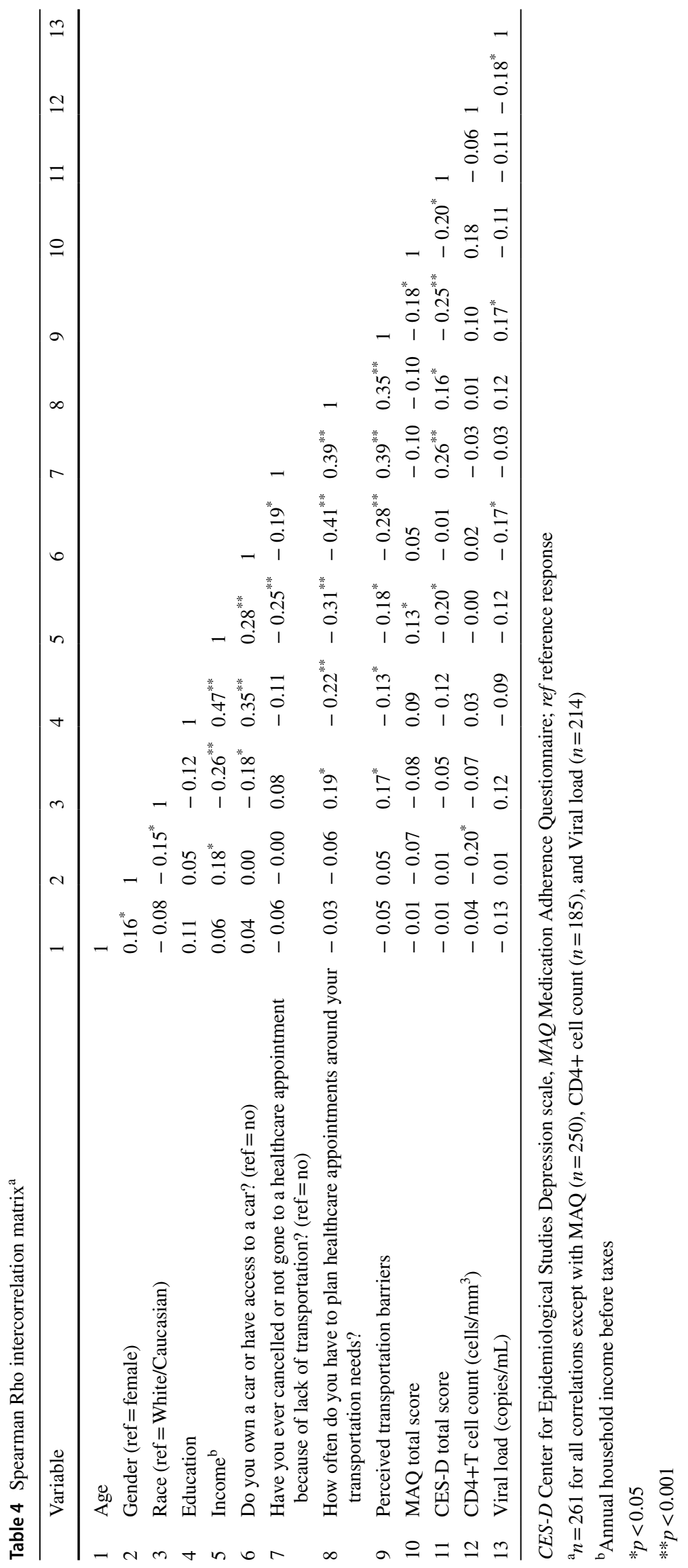


barriers were not significantly associated can be found in Supplemental Tables S1-S6). For every unit increase in perceived transportation barriers, there was an associated 4.59point $(95 \% \mathrm{CI}-7.97,-1.21)$ reduction in general health perceptions, 5.41-point (95\% CI $-9.32,-1.50)$ reduction in pain, 4.37 -point $(95 \% \mathrm{CI}-8.34,-0.39)$ reduction in social functioning, 3.59-point $(95 \% \mathrm{CI}-6.84,-0.34)$ reduction in health distress, and 4.36-point (95\% CI - 7.69, - 1.03) reduction in health transitions. Greater depressive symptoms were associated with worse functioning across all health domains, whereas HIV and demographic variables were not statistically significant in the context of both depression and perceived transportation barriers.

\section{Discussion}

Previous research has established an association between treatment outcomes, such as initiation and retention to HIV care and transportation barriers in PWH $[6,9,15,16]$, yet lacking is an understanding of how transportation barriers may be associated with HRQOL, a known indicator of well-being and mortality in older adults [33, 34]. Given this empirical gap, the current study investigated the association between perceived transportation barriers and health-related outcomes including HRQOL in a sample of middle-aged and older PWH. We found partial support for our hypotheses as greater perceived transportation barriers (defined as how much of a problem it was for an individual to access the care, services, or opportunities they wished to obtain because of a lack of transportation) was associated with lower scores of general health perceptions, pain, social functioning, health distress, and health transitions over the previous four weeks independent of HIV disease characteristics, socioeconomic indicators, and depressive symptoms.

Outside of HRQOL, perceptions of transportation barriers were also associated with both objective and self-reported health outcomes, including medication adherence, depressive symptoms, and viral load. Additionally, consistent with previous literature, greater perceived transportation barriers were associated with indicators of HIV care retention, such as missing or cancelling a healthcare appointment and having to more frequently plan healthcare appointments around transportation needs. While examining differences in health outcomes between transportation barriers and contributors was not the focus of the current study, there was a notable lack of association between ownership or access to a vehicle and medication adherence, depressive symptoms, and HRQOL (data not presented). While this could be a product of how transportation barriers were measured in the current study, Yehia et al. noted similar findings using a sample of PWH from Ryan White-funded clinics in the Northeast where public transportation options are more readily

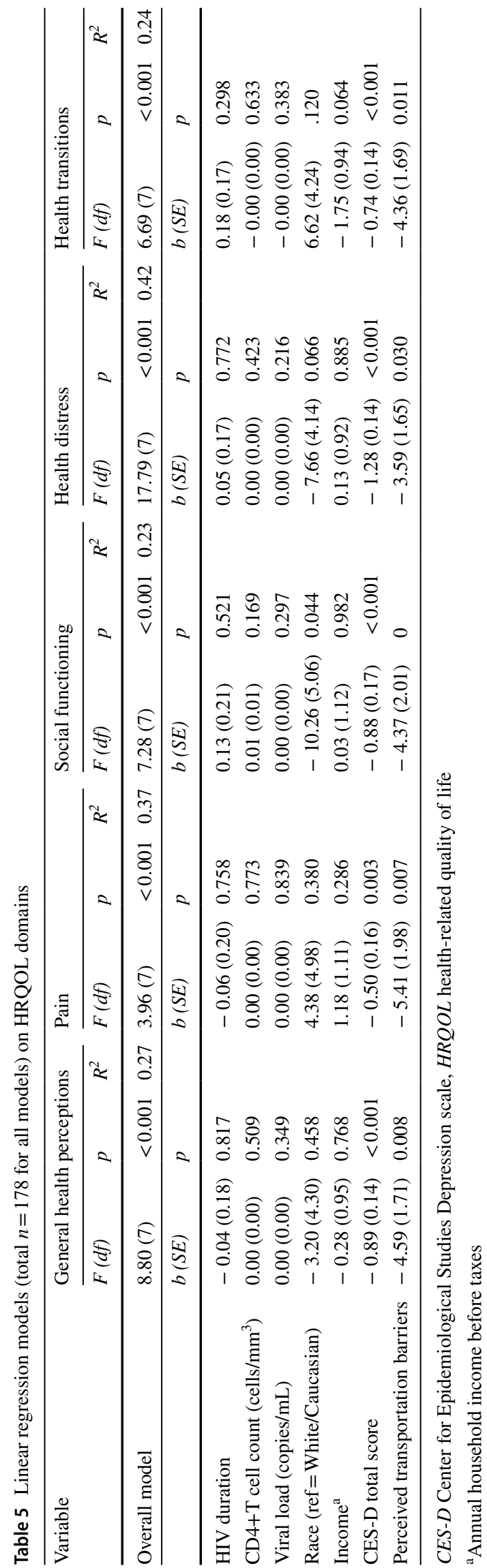


accessible, and services or alternatives were provided by the clinic [9]. From these findings the authors concluded that, although transportation alternatives (in some form) are provided by clinics, they still may not fully mitigate barriers such as unreliable shuttle van services, inclement weather, heavy traffic, and living in areas with limited accessibility [9]. Chaiyachati et al. also found when conducting a randomized control trial using rideshare services (e.g., Lyft) in the Northeast as an alternative to providing nonemergency medical transportation (NEMT) to Medicaid beneficiaries, that when the rideshare service was offered, not all participants were interested even when help with scheduling the service was provided; additionally it did not improve their outcome of interest, missed appointment rates, compared to the control arm who were not offered rideshare services [35]. In the U.S., rideshare services have become a more popular and potential cheaper (compared to other forms of NEMT [35]) alternative to driving personally owned vehicles; however, limited knowledge of how the service operates [36], inaccessibility for those with mobility issues or disabilities [37], and residing outside of urban, metropolitan areas [38] may contribute to lack of interest or low usage of rideshare services within older populations.

Additionally, as one of the current study aims was to examine the association between perceived transportation barriers to care and HRQOL, what remains uncertain is the directionality or temporality of these associations. For example, still unknown is how transportation barriers may lower HRQOL (e.g., pain or social functioning) and what mechanisms (e.g., depression) may contribute to the worsening of disease management behaviors over time. Understanding of this directionality could be useful for future interventions and strategies when assessing barriers to treatment, health promotion, and overall health and well-being. Transportation barriers may differ across individuals and situations as they can present as a long-lasting problem (e.g., chronic medical condition) or be the result of a temporary event (e.g., a car breaks down, suspension of a license). Further research is needed to understand how the presence and chronicity of the issue contributes to overall mental and physical health in samples of PWH. Regardless of the directionality, it can be interpreted that those who are the most vulnerable (e.g., those with poorer health perceptions, greater pain, smaller social networks, greater health distress, and worsening health conditions) are also the ones with difficulty accessing reliable transportation to necessary healthcare appointments.

\section{Considerations for Healthcare and Traffic Safety}

Healthcare providers are encouraged to assess transportation barriers in this vulnerable, aging population as access to reliable transportation is a key social determinant of health that promotes health equity. Whereas reliable and adequate transportation is important for all individuals, it is especially important for individuals who have never learned how to drive, are medically unfit to drive, lack the financial means to drive, or reside in geographic areas with underdeveloped public transportation. For example, in samples of PWH, rurality has been cited as a major issue that is intertwined with transportation barriers $[6,14,15]$. While most of the current study sample resided in urban or metropolitan areas within the Deep South (determined by provided zip codes), there were still notable perceptions of transportation barriers, which was associated with their HRQOL. These perceptions could be potentially magnified for rural areas that require longer commutes, as shown in studies with AfricanAmerican women living in rural Alabama [6, 39].

Furthermore, McManus et al. conducted a national survey with HIV clinicians on their knowledge and attitudes towards the Affordable Care Act expansion, which included coverage for NEMT [40]. Of the HIV clinicians who responded, all reported transportation as a top barrier in both states with and without Medicaid expansion, although transportation barriers were higher in non-expansion states (47\% vs. 29\%) and in the Midwest, South, and West compared to the Northeast. Unknown from their study was how rurality contributed to these findings or if their patients also saw transportation as a major barrier to care [40]. Additionally, when healthcare providers are assessing transportation barriers, social support and networks should be assessed as cultural factors and HIV/AIDS stigma [5, 6] may also extend to transportation barriers for older PWH. For example, previous research has shown that, while narrowing, there is still a persisting gender difference in driving habits and cessation within older adults, with women enacting in driving cessation earlier in life than men [41, 42]. Also, research has shown evidence of increased prevalence or burden of neurocognitive deficits in those aging with HIV (e.g., HIV-associated Neurocognitive Disorder [HAND]) which can potentially impact driving abilities [43, 44]. Over time, as individuals age with HIV and mobility needs change, they may become more reliant on family members or social networks for transportation, opening a discussion and dialog of HIV-status disclosure. Likewise, many PWH as they age will likely become caregivers themselves [45] and will have to coordinate transportation for their loved ones and family members while also managing their own mobility needs.

The COVID-19 (SARS-CoV-2) pandemic has brought to light many social inequities in healthcare [46] and transportation [47], along with patient care options for maintaining communication and outreach with at-risk patients. These solutions, in some form, may also benefit PWH with known transportation barriers and those residing in rural areas, individuals who may also have been disproportionately impacted by the COVID-19 pandemic (i.e., compounded inequity). For example, the National Institutes of Health 
Office of AIDS Research has provided interim guidance for providing care for PWH during COVID-19 [48], such as maintaining an adequate supply of ART medications by verifying that the patient has an adequate supply of all necessary medications and that refills are expedited when needed. To that extent, exploring mail-order delivery options with participants rather than in-person medication pick up may also help alleviate burden from transportation barriers. Healthcare providers are also encouraged to offer telehealth or virtual visits for routine or non-urgent care, but also to be mindful of assessing worsening mental health symptoms as well as increased risk for intimate partner violence as both are associated with increased social isolation and poor retention in HIV care [49].

Along with healthcare providers, there are also implications for transportation policy makers to consider in terms of adapting and updating traffic safety policy, infrastructure, and public transit to reduce systemic barriers to reliable transportation. For example, recent research has shown evidence of linkages between non-driving-related licensure suspensions and vulnerable neighborhoods [50, 51]. Joyce et al. found, using linked data from New Jersey, that over a 15-year period, more non-driving-related licensure suspensions (e.g., failure to appear in court or pay a court fee for drug-related convictions) were found in census tracts with higher proportions of Black and Hispanic residents, higher unemployment rates, and lower household median incomes [50]. Still unknown is how these licensure suspensions translate to transportation barriers and health-related outcomes. While the current study was not designed to assess this granular level of association in the context of transportation barriers, within our study sample only $63.6 \%(n=166)$ reported having a current and valid driver's license, yet $81.6 \%$ $(n=213)$ were current drivers regardless of licensure status. Additionally, of the 95 who did not have a current license, $46.3 \%(n=44)$ attributed the reason of licensure suspension to unpaid tickets, driving while under the influence, or not having the financial means for reinstatement (qualitative data not presented). More research is needed to assess the reasons for non-licensure in clinical samples such as HIV/AIDS and how it may contribute to transportation barriers and negative health outcomes. Additionally, policy makers and stakeholders should take into consideration how they can identify and eliminate structural inequities within transportation systems for vulnerable populations such as those aging with HIV and other chronic diseases using public health paradigms such as Critical Race Theory [52], policy design approaches such as Complete Streets (see review by Jordan and Ivey [53]), and advocating for increased public transportation funding and what types of trip destinations can be considered NEMT [47]. By acknowledging factors such as historical context, structural determinism, and social location through community engagement strategies, future transportation needs can be addressed to help remedy transportation barriers for those most at-risk [52, 54].

\section{Strength and Limitations}

A major strength of the current study was the use of a standardized HRQOL screener, MOS-HIV [29], that has been well validated for HIV/AIDS samples and is comparable to other validated HRQOL screeners such as the SF-36 [28-31]. Additionally, the study focused on the Deep South, a geographical region of the U.S. that is disproportionally affected by the HIV/AIDS epidemic $[4,5]$ that is also less likely to have alternative forms of public transportation or transit $[6,39]$ and combined with high burdens of socioeconomic disparity [5]. As with all studies, there are limitations. For example, there are many potential causes of transportation barriers across individuals; therefore, the questions used here to measure transportation barriers may not reflect the overall breadth of issues with adequate and reliable transportation. Another limitation is that we were unable to obtain a more granular level of residence, such as census tracks, to provide a better index of rurality, walkable communities, or neighborhood-level disadvantage that would be more informative over zip codes which cover large areas of residence or rurality. Lastly, the majority of the sample were on ART and had undetectable viral load, suggesting the sample was largely individuals with well controlled HIV and may not be generalizable to other samples of PWH in the Deep South, such as those who are not currently in long-term treatment. Given retention to care correlates with overall HIV/AIDS disease management, the overall findings may be conservative in representing the level of transportation barriers for PWH who are not on ART, have unmanaged HIV/AIDS, and experience worse disease-related outcomes. Importantly, while there was missing clinic data for HIV/ AIDS clinical values, there were no significant differences seen for sociodemographics between those with missing and non-missing clinic data.

\section{Conclusions}

Using a sample of middle-aged and older PWH from the Deep South, the association between perceived transportation barriers and HRQOL was examined. Individuals with poor HRQOL, such as those with greater pain or health distress, may also be the individuals who are most vulnerable in terms of inadequate access to reliable transportation to access healthcare appointments. Implications involve a thorough healthcare assessment of transportation options and barriers when working with HIV/AIDS clinical samples along with further discussion into how public health 
approaches can be integrated into traffic safety policy and design to reduce systemic inequities that contribute to transportation barriers and health-related outcomes.

Supplementary Information The online version contains supplementary material available at https://doi.org/10.1007/s10461-021-03560-x.

Acknowledgements A special thank you to the participants, the UAB Center for Research on Applied Gerontology, UAB School of Nursing, UAB Department of Psychology, UK Department of Health, Behavior \& Society, and UK Graduate Center for Gerontology.

Author Contributions All authors contributed to the study conception and design. Material preparation and data collection were performed by $\mathrm{PF}$ and DV. Data preparation and analysis was performed by $\mathrm{CP}$ and $\mathrm{PF}$. The first draft of the manuscript was written by $\mathrm{CP}$ and all authors commented on previous versions of the manuscript. All authors read and approved the final manuscript.

Funding This study was supported in part by a National Institutes of Health (NIH)/National Institute of Mental Health (NIMH) R01-award (R01 MH106366- 01A1; ClinicalTrials.gov; NCT02758093; An RCT of Speed of Processing Training in Middle-aged and Older Adults with HIV; PI: Vance); NIH/National Institute on Aging (NIA) R00-award (R00 AG048762; A Novel Neurorehabilitation Approach for Cognitive Aging with HIV; PI: Fazeli); NIH/NIA P30-award (Edward R. Roybal Center for Translational Research in Aging and Mobility; P30 AG022838; PI: Ball), NIH/NIMH R25-award (R25 MH108389; Sustained Training on Aging and HIV Research; PIs: Jeste, Letendre], ORWH and NIH/NIDA BIRCWH grant (\#5K12DA035150; PI: Curry); and the U.S. Department of Transportation Dwight D. Eisenhower Graduate Fellowship Program (PI: Pope).

\section{Availability of Data and Material N/A}

Code Availability N/A.

\section{Declarations}

Conflict of interest Authors have no known conflict of interest to disclose.

Ethical Approval The study procedure was reviewed and approved by the University of Alabama at Birmingham's Institutional Review Board.

Consent to Participate All participants provided written informed consent and were compensated for participating.

Consent for Publication N/A.

\section{References}

1. Centers for Disease Control and Prevention (CDC). HIV in the Southern United States 2019.

2. Centers for Disease Control and Prevention (CDC). HIV Surveillance Report, 2018 updated.
3. Centers for Disease Control and Prevention (CDC). HIV in the United States by Region. 2021; https://www.cdc.gov/hiv/statistics/ overview/geographicdistribution.html. Accessed 032021

4. Watson M, Johnson SD, Zhang T, Oster AM. Characteristics of and trends in HIV diagnoses in the Deep South region of the United States, 2012-2017. AIDS Behav. 2019;23(3):224-32.

5. Reif S, Safley D, McAllaster C, Wilson E, Whetten K. State of HIV in the US Deep South. J Commun Health. 2017;42(5):844-53.

6. Kempf M-C, McLeod J, Boehme AK, et al. A qualitative study of the barriers and facilitators to retention-in-care among HIVpositive women in the rural southeastern United States: implications for targeted interventions. AIDS Patient Care STDs. 2010;24(8):515-20.

7. Syed ST, Gerber BS, Sharp LK. Traveling towards disease: transportation barriers to health care access. J Commun Health. 2013;38(5):976-93.

8. Butkus R, Rapp K, Cooney TG, Engel LS. Envisioning a better US health care system for all: reducing barriers to care and addressing social determinants of health. Ann Intern Med. 2020;172:S50-9.

9. Yehia BR, Stewart L, Momplaisir F, et al. Barriers and facilitators to patient retention in HIV care. BMC Infect Dis. 2015;15(1):246.

10. O'Neill D, Walshe E, Winston F. Transportation equity, health, and aging: A novel approach to healthy longevity with benefits across the life span. NAM Perspect. 2019.

11. Resurrección DM, Motrico E, Rigabert A, et al. Barriers for nonparticipation and dropout of women in cardiac rehabilitation programs: a systematic review. J Womens Health. 2017;26(8):849-59.

12. Harrison AL, Hunter EG, Thomas H, Bordy P, Stokes E, Kitzman P. Living with traumatic brain injury in a rural setting: supports and barriers across the continuum of care. Disabil Rehabil. 2017;39(20):2071-80.

13. Ryvicker M, Bollens-Lund E, Ornstein KA. Driving status and transportation disadvantage among medicare beneficiaries. J Appl Gerontol. 2020;39(9):935-43.

14. Heckman TG, Somlai AM, Peters J, et al. Barriers to care among persons living with HIV/AIDS in urban and rural areas. AIDS Care. 1998;10(3):365-75.

15. Reif S, Golin CE, Smith SR. Barriers to accessing HIV/AIDS care in North Carolina: rural and urban differences. AIDS Care. 2005;17(5):558-65.

16. Lopes BLW, Eron JJ Jr, Mugavero MJ, Miller WC, Napravnik S HIV care initiation delay among rural residents in the Southeastern United States, 1996 to 2012. J Acquir Immune Defic Syndr. 2017;76(2):171-6.

17. Centers for Disease Control and Prevention (CDC). HealthRelated Quality of Life (HRQOL). 2018; https://www.cdc.gov/ hrqol/concept.htm.

18. Mrus JM, Williams PL, Tsevat J, Cohn SE, Wu AW. Gender differences in health-related quality of life in patients with HIV/ AIDS. Qual Life Res. 2005;14(2):479-91.

19. Worthington C, Krentz HB. Socio-economic factors and healthrelated quality of life in adults living with HIV. Int J STD AIDS. 2005;16(9):608-14.

20. Call SA, Klapow JC, Stewart KE, et al. Health-related quality of life and virologic outcomes in an HIV clinic. Qual Life Res. 2000;9(9):977-85.

21. Rodriguez-Penney AT, Iudicello JE, Riggs PK, et al. Co-morbidities in persons infected with HIV: increased burden with older age and negative effects on health-related quality of life. AIDS Patient Care STDS. 2013;27(1):5-16.

22. Erskine NA, Gandek B, Tran HV, et al. Barriers to healthcare access and to omprovements in health-related quality of life after an Acute Coronary Syndrome (from TRACE-CORE). Am J Cardiol. 2018;122(7):1121-7. 
23. Vance DE, Fazeli PL, Shacka J, et al. Testing a computerized cognitive training protocol in adults aging with HIV-associated neurocognitive disorders: randomized controlled trial rationale and protocol. JMIR Res Protoc. 2017;6(4):e68-e68.

24. USDA Economic Research Service. Rural-urban communiting area codes. 2020; https://www.ers.usda.gov/data-products/ruralurban-commuting-area-codes/. Accessed 3/2021.

25. Wilson IB, Lee Y, Michaud J, Fowler FJ Jr, Rogers WH. Validation of a new three-item self-report measure for medication adherence. AIDS Behav. 2016;20(11):2700-8.

26. Radloff LS. The CES-D Scale: a self-report depression scale for research in the general population. Appl Psychol Meas. 1977; 1:385-401.

27. Lewinsohn PM, Seeley JR, Roberts RE, Allen NB. Center for Epidemiologic Studies Depression Scale (CES-D) as a screening instrument for depression among community-residing older adults. Psychol Aging. 1997;12(2):277-87.

28. Wu AW, Rubin HR, Mathews WC, et al. A health status questionnaire using 30 items from the Medical Outcomes Study. Preliminary validation in persons with early HIV infection. Med Care. 1991;29(8):786-98.

29. Cooper V, Clatworthy J, Harding R, Whetham J. Measuring quality of life among people living with HIV: a systematic review of reviews. Health Qual Life Outcomes. 2017;15(1):220.

30. Revicki DA, Sorensen S, Wu AW. Reliability and validity of physical and mental health summary scores from the Medical Outcomes Study HIV Health Survey. Med Care. 1998;36(2):126-37.

31. Wu AW, Revicki DA, Jacobson D, Malitz FE. Evidence for reliability, validity and usefulness of the Medical Outcomes Study HIV Health Survey (MOS-HIV). Qual Life Res. 1997;6(6):481-93.

32. IBM SPSS Statistics for Windows [computer program]. Version 27.0. Armonk: IBM Corp.; 2020.

33. Brown DS, Thompson WW, Zack MM, Arnold SE, Barile JP. Associations between health-related quality of life and mortality in older adults. Prev Sci. 2015;16(1):21-30.

34. Upton D, Upton P. Quality of life and well-being. In: Upton D, Upton P, editors. Psychology of wounds and wound care in clinical practice. Cham: Springer International Publishing; 2015. p. 85-111.

35. Chaiyachati KH, Hubbard RA, Yeager A, et al. Association of rideshare-based transportation services and missed primary care appointments: a clinical trial. JAMA Intern Med. 2018;178(3):383-9.

36. Vivoda JM, Harmon AC, Babulal GM, Zikmund-Fisher BJ. E-hail (rideshare) knowledge, use, reliance, and future expectations among older adults. Transp Res Part F Traffic Psychol Behav. 2018;55:426-34.

37. Dickerson AE, Molnar LJ, Bedard M, et al. Transportation and aging: an updated research agenda to advance safe mobility among older adults transitioning from driving to non-driving. Gerontologist. 2019;59(2):215-21.

38. Choi M, Schuster AM, Schoenberg NE. Solutions to the challenge of meeting rural transportation needs: middle-Aged and older adults' perspectives. J Gerontol Soc Work. 2019;62(4):415-31.

39. Moneyham L, McLeod J, Boehme A, et al. Perceived barriers to HIV care among HIV-infected women in the Deep South. J Assoc Nurses AIDS Care. 2010;21(6):467-77.
40. McManus KA, Ferey J, Farrell E, Dillingham R. National survey of US HIV clinicians: Knowledge and attitudes about the Affordable Care Act and opinions of its impact on quality of care and barriers to care. Open Forum Infect Dis. 2020;7(7):ofaa225.

41. Bauer MJ, Adler G, Kuskowski MA, Rottunda S. The influence of age and gender on the driving patterns of older adults. J Women Aging. 2003;15(4):3-16.

42. Hakamies-Blomqvist L, Siren A. Deconstructing a gender difference: driving cessation and personal driving history of older women. J Safety Res. 2003;34(4):383-8.

43. Vance DE, Fazeli PL, Ball DA, Slater LZ, Ross LA. Cognitive functioning and driving simulator performance in middleaged and older adults with HIV. J Assoc Nurses AIDS Care. 2014;25(2):e11-26.

44. Marcotte TD, Heaton RK, Wolfson T, et al. The impact of HIVrelated neuropsychological dysfunction on driving behavior. J Int Neuropsychol Soc. 1999;5(7):579-92.

45. Lee Y, Batey DS, Clay OJ, Emlet CA, Fazeli PL, Vance DE. Older caregivers with HIV: an unrecognized gap in the literature. J Assoc Nurses AIDS Care. 2021;32(1):29-36.

46. Baptiste D-L, Commodore-Mensah Y, Alexander KA, et al. COVID-19: shedding light on racial and health inequities in the USA. J Clin Nurs. 2020;29(15-16):2734-6.

47. Chen KL, Brozen M, Rollman JE, et al. How is the COVID-19 pandemic shaping transportation access to health care? Transp Res Interdiscip Perspect. 2021;10:100338.

48. Office of AIDS Research [OAR]. Interim Guidance for COVID-19 and Persons with HIV. 2021; https://clinicalinfo.hiv.gov/en/guide lines/covid-19-and-persons-hiv-interim-guidance/interim-guida nce-covid-19-and-persons-hiv. Accessed 08/2021.

49. Siemieniuk RAC, Krentz HB, Gill MJ. Intimate partner violence and HIV: a review. Curr HIV/AIDS Rep. 2013;10(4):380-9.

50. Joyce NR, Pfeiffer MR, Zullo AR, Ahluwalia J, Curry AE. Individual and geographic variation in Driver's license suspensions: evidence of disparities by race, ethnicity and income. J Transp Health. 2020;19:100933.

51. Joyce NR, Zullo AR, Ahluwalia JS, Pfeiffer MR, Curry AE. Driver's license suspension policies as a barrier to health care. Am J Public Health. 2019;109(12):1692-3.

52. Ford CL, Airhihenbuwa CO. Critical race theory, race equity, and public health: toward antiracism praxis. Am J Public Health. 2010;100:S30-5.

53. Jordan SW, Ivey S. Complete streets: promises and proof. J Urban Plan Dev. 2021;147(2):04021011.

54. Ingram M, Leih R, Adkins A, Sonmez E, Yetman E. Health disparities, transportation equity and complete streets: a case study of a policy development process through the lens of critical race theory. J Urban Health. 2020;97(6):876-86.

Publisher's Note Springer Nature remains neutral with regard to jurisdictional claims in published maps and institutional affiliations. 